\title{
Haemodynamics to predict outcome in pulmonary hypertension due to left heart disease: a meta-analysis
}

\author{
To the Editor:
}

Pulmonary hypertension $(\mathrm{PH})$ is a common complication of left heart disease (LHD), as the result of a "passive" increase of left atrial pressure (LAP), leading to isolated post-capillary PH [1-3]. Several haemodynamic parameters have been proposed to identify a more severe phenotype of PH-LHD, for which the increase in pulmonary artery pressure cannot be accounted for by the increase in LAP, and described as combined post-capillary $\mathrm{PH}$ with a pre-capillary component, or $\mathrm{CpcPH} \mathrm{[1-3].} \mathrm{The} \mathrm{latter}$ haemodynamic phenotype may potentially expose patients to a higher risk of right ventricular failure and a poorer outcome $[4,5]$. However, since the last World Symposium in 2013, how to define the pre-capillary component in PH-LHD has been a matter of debate, as pathophysiological arguments have been suggested to potentially contrast with clinical and prognostic evidences [1].

Given current controversies and uncertainties on this topic, we sought to perform a meta-analysis in order to determine which variable would be associated with mortality in PH-LHD.

We conducted a Medline literature research according to PRISMA recommendations [6] on articles published since 2013, when the diastolic pressure gradient (DPG) was introduced as a possible marker of the pre-capillary component in PH-LHD [2]. We used the following combinations of search terms ("pulmonary hypertension" OR "heart failure" OR "left heart disease") AND ("prognosis" OR "outcome" OR "outcomes" OR "survival”) AND ("pulmonary vascular resistance” OR "diastolic pressure gradient" OR "diastolic pressure difference" OR "diastolic pulmonary gradient" OR "diastolic pulmonary vascular pressure gradient" OR "pulmonary artery compliance" OR "hemodynamics”). We only considered papers in which DPG and/or pulmonary vascular resistance (PVR) and/or pulmonary artery compliance (PAC) were analysed for their capability to predict survival in PH-LHD. We excluded articles reporting composite outcome, those focusing only on outcome after heart transplantation, evaluating short-term follow-up, or including unstable patients. PRISMA flow diagram is shown in figure 1a.

Out of 32 articles, 10 responded to the above-mentioned criteria [4, 5, 7-14]. These articles considered DPG, PVR and PAC as continuous or dichotomous variables. For purpose of consistency, and to better individuate the risk associated with each variable, independently of arbitrary cut-offs, we only included those that reported the prognostic power of variables intended in a continuous way. The final analysis was thus conducted on six articles [4, 7-11], including 2513 patients with LHD overall, followed up for 9-15 years. In four studies, all patients had $\mathrm{PH}[4,8,9,11]$, while in the remaining two articles at least two-thirds of patients had PH [7, 10]. In the majority of the studies, a multivariate analysis was performed, adjusting the hazard ratios associated with PVR, DPG and PAC for covariates such as age and sex $[4,8,10,11]$, body mass index $[10,11]$, ethnicity $[8,11]$, and also for comorbidities $[4,7]$ and other haemodynamic parameters $[8,10]$.

Analysis of the different populations revealed heterogeneity in terms of age, gender and aetiology of PH-LHD. Mean age was 60 years old, with mean values varying between 49 and 69 years old across the studies. Younger ages were more represented in heart failure with reduced ejection fraction (HFrEF) cohorts while older ages were found in cohorts of heart failure with preserved ejection fraction (HFpEF) or valvular heart disease. Male sex prevalence varied between 25 and 79\%. HFpEF was the leading aetiology

@ERSpublications

PVR, DPG, PAC are all associated with survival in pulmonary hypertension due to left heart disease http://ow.ly/R8sF30ieHSQ

Cite this article as: Caravita S, Dewachter C, Soranna D, et al. Haemodynamics to predict outcome in pulmonary hypertension due to left heart disease: a meta-analysis. Eur Respir J 2018; 51: 1702427 [https:// doi.org/10.1183/13993003.02427-2017]. 
a)

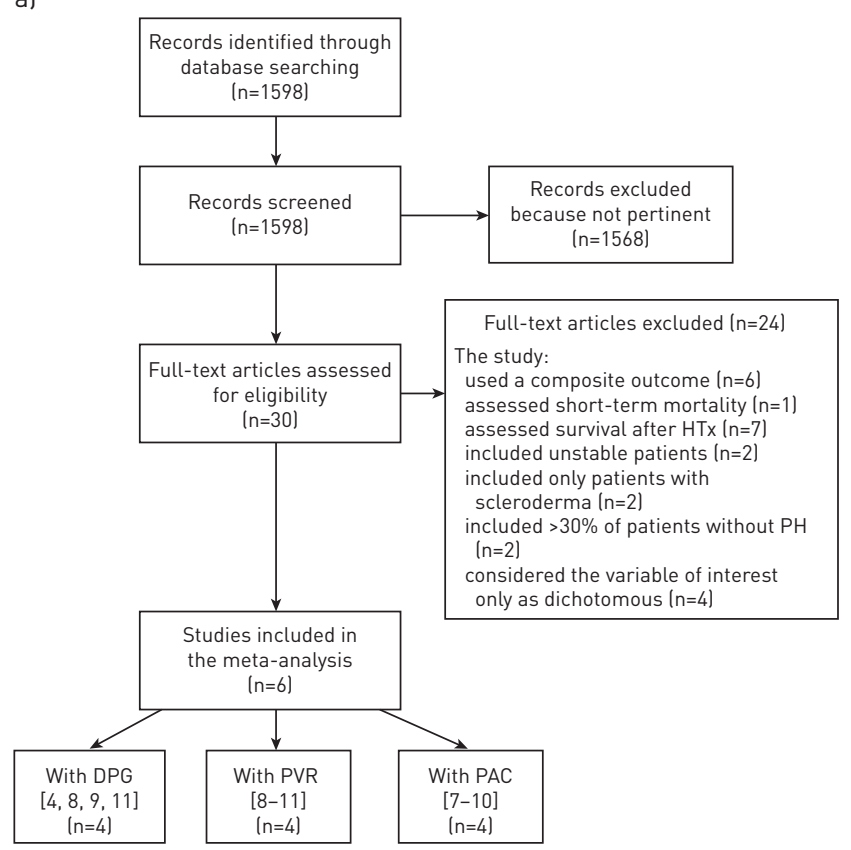

b)

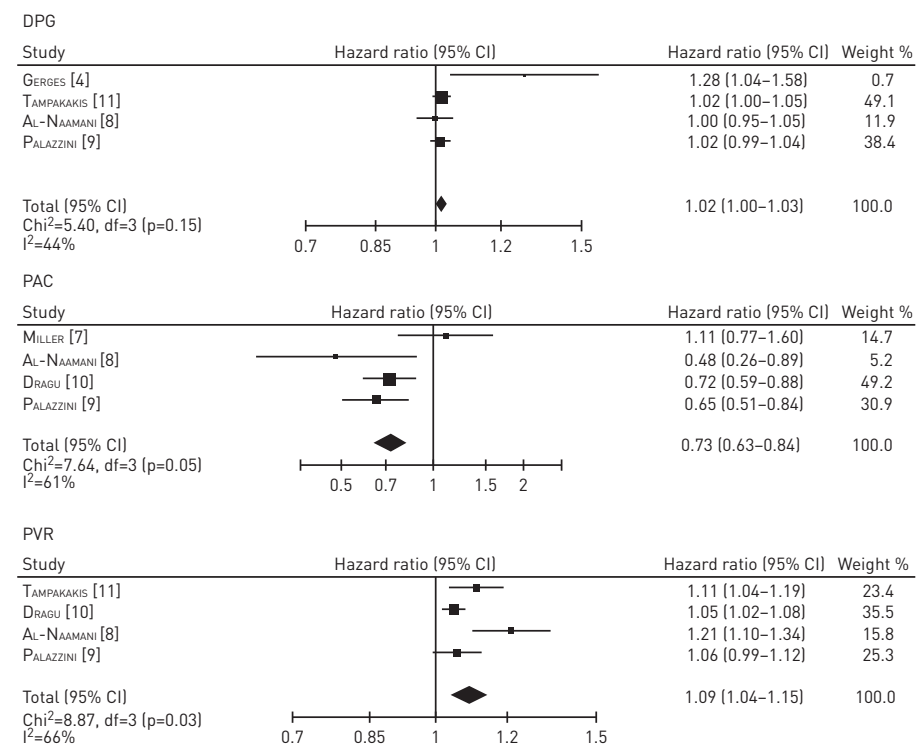

FIGURE 1 a) Flow diagram of study selection. b) Forest plot analysis for survival according to pulmonary vascular resistance (PVR), diastolic pressure gradient (DPG) and pulmonary arterial compliance (PAC). HTx: heart transplant; PH: pulmonary hypertension.

in 3 studies $[4,8,10]$, while valvular heart disease was the leading aetiology or a significant comorbidity (present in up to $30 \%$ of patients) in three studies $[4,7,9]$. In these latter studies, it is possible that right heart catheterisation has been performed in some patients as a pre-therapeutic assessment, although this has not been systematically reported by the authors. Neither symptoms (e.g. New York Heart Association functional class) nor concomitant relevant pathological conditions were consistently reported in all studies.

Mean pulmonary artery pressure was on average $35 \mathrm{mmHg}$, mean PVR 3.0 WU, mean DPG was $1.2 \mathrm{mmHg}$ (this latter derived from five studies $[4,8-11]$ ) and mean PAC $2.5 \mathrm{~mL} \cdot \mathrm{mmHg}^{-1}$ (derived from four studies [7-10]). Mean PVR was $>3 \mathrm{WU}$ in three studies [7-9], mean DPG was $<7 \mathrm{mmHg}$ in all studies, while mean PAC was $<2.3 \mathrm{~mL} \cdot \mathrm{mmHg}^{-1}$ in two studies $[8,9]$. Based on mean $\pm \mathrm{SD}$ values, there is a high suspicion of a significant proportion of negative DPGs, at least in five out of six studies [4, 8-11].

We pooled the original estimates by using both the fixed-effects model and the random-effects model. Heterogeneity between study-specific estimates was tested using the $\mathrm{Q}$ statistic and $\mathrm{I}^{2}$ index. When a significant heterogeneity was found $(\mathrm{Q}$ statistic $\mathrm{p}$-value $<0.05)$, the results from the random-effects model were presented. To evaluate publication bias, the "trim and fill" method was used [15]. This is an extension of the funnel plot technique, where "missing studies" are identified and estimated based on the symmetry of the funnel plot [16].

Forest plots analysis for survival according to DPG, PVR and PAC are depicted in figure 1b. Increasing values of PVR and DPG were associated with higher risk of death in PH-LHD, while increasing values of PAC were associated with lower risk. Results did not change when performing a sensitivity analysis including only the four studies in which all patients had $\mathrm{PH}[4,8,9,11]$. Some suggestions of presence of publication bias come from the results obtained from the "trim and fill" method. The summary estimates coming from this method are very similar to the summary original estimates for each outcome, even if the summary estimates of DPG and PAC were not statistically significant. Nevertheless, the paucity of included studies puts caution in interpreting the results obtained by the "trim and fill" method [16].

This meta-analysis may thus reconcile contrasting theories and evidences on which parameter(s) to define a pre-capillary component and/or to predict prognosis in PH-LHD.

Indeed, the DPG was re-introduced as a marker of disease in 2013 based on solid physiological background, histological demonstration of association with pulmonary arterial remodelling, and possible prognostic potential $[2,4]$. However, the DPG is a small number highly subjected to background noise, partly related to problems in the measurement of diastolic pulmonary artery pressure and pulmonary artery wedge pressure (PAWP) [1, 17], so that over $30 \%$ of patients in the published series may present 
with negative DPG, which is deemed to be physiologically impossible [1]. This may be due to technical limitations and interpretations drawbacks in the accuracy of PAWP and/or diastolic pulmonary artery pressure determination [1-3, 17], that may also affect, to some extent, PAC and PVR [1]. It is also interesting to note that only a minority of patients with PH-LHD may present with high DPGs, which is confirmed by low mean DPGs values (about $1 \mathrm{mmHg}$ ) in the whole cohort that was subjected to meta-analysis. However, at variance from some individual reports, DPG was found to be associated with outcome, with a $2 \%$ increase in risk for a unitary increase in DPG.

Based on contrasting evidences on DPG, PVR was reintroduced because it may have additional value as an indirect reflection of right ventricular function [3] since, in patients with PH-LHD, it may normalise when improving cardiac output by means of systemic vasodilators or inotropes. It should be underscored that PVR is a well-consolidated and longstanding used variable in clinical practice, despite physiological limitations due to its flow and filling pressure dependency [1]. Our meta-analysis confirms available data on the prognostic discriminative potential of PVR. A unitary increase in PVR was associated with $9 \%$ increase in risk of adverse outcome.

Recently, PAC has been increasingly investigated in PH-LHD, and it was proposed as a predictor of outcome [1,7-10]. However, PAC is an even smaller number than DPG, it may be highly dependent on PAWP [18], and the formula utilised to calculate it in clinical practice may overestimate the true PAC by 60 to $80 \%$ [1]. Despite these limitations, PAC is also associated with outcome, with higher values being related with lower risk.

Some other limitations of this analysis should be underlined. In particular, only few retrospective studies (or retrospective analysis of a prospective registry) were available for this analysis, without prospective validation of haemodynamic measurements. This should encourage confirmation of our results in ad hoc designed prospective studies.

In summary, the present analysis confirms that the population of PH-LHD is heterogeneous in terms of age, aetiology, proportion of negative DPGs and haemodynamic presentation. However, despite the differences in cohorts and the intrinsic limitations of each variable [1], PVR, DPG and PAC appear to be associated with survival in PH-LHD. Importantly, we may speculate that these variables should be viewed as complementary and may be used in combination as an intrinsic control. Which combination of variables would best be used to predict outcome remains a matter of debate.

Sergio Caravita ${ }^{1,2}$, Céline Dewachter ${ }^{1}$, Davide Soranna ${ }^{2,3}$, Sandy Carolino D’Araujo ${ }^{1}$, Amina Khaldi ${ }^{1}$, Antonella Zambon ${ }^{2,3}$, Gianfranco Parati ${ }^{2,4}$, Antoine Bondue ${ }^{1}$ and Jean-Luc Vachiéry ${ }^{1}$

${ }^{1}$ Dept of Cardiology, Cliniques Universitaires de Bruxelles, Hôpital Académique Erasme, Brussels, Belgium. ${ }^{2}$ Dept of Cardiovascular, Neural and Metabolic Sciences, Ospedale S. Luca IRCCS Istituto Auxologico Italiano, Milan, Italy. ${ }^{3}$ Dept of Statistic and Quantitative Methods, University of Milano-Bicocca, Milan, Italy. ${ }^{4}$ Dept of Medicine and Surgery, University of Milano-Bicocca, Milan, Italy.

Correspondence: Jean-Luc Vachiéry, Dept of Cardiology, CUB Hôpital Erasme, 808 Route de Lennik, 1070 Brussels, Belgium. E-mail: jeanluc.vachiery@erasme.ulb.ac.be

Received: Nov 232017 | Accepted after revision: Jan 232018

Support statement: S. Caravita is the recipient of a ERS PAH Short-Term Research Training Fellowship (STRTF 2014-5264) supported by an unrestricted grant by GSK, and of the international grant "Cesare Bartorelli" for the year 2014 funded by the Italian Society of Hypertension. J-L. Vachiery is the holder of the Actelion Research Chair on Pulmonary Hypertension in his department.

Conflict of interest: J-L. Vachiéry has received grants from Actelion, outside the submitted work. S. Caravita has received a travel grant from Actelion, outside the submitted work.

Acknowledgements: The authors would like to thank Irene Lang, Christian Gerges and Mario Gerges (Division of Cardiology, Department of Internal Medicine II, General Hospital Vienna (AKH-Wien), Medical University of Vienna, Vienna, Austria) for having provided their own data, otherwise not available for this meta-analysis.

\section{References}

1 Naeije R, Gerges M, Vachiéry JL, et al. Hemodynamic phenotyping of pulmonary hypertension in left heart failure. Circ Heart Fail 2017; 10: e004082.

2 Vachiéry JL, Adir Y, Barberà JA, et al. Pulmonary hypertension due to left heart diseases. J Am Coll Cardiol 2013; 62: D100-D108.

3 Galiè N, Humbert M, Vachiery JL, et al. 2015 ESC/ERS Guidelines for the diagnosis and treatment of pulmonary hypertension: The Joint Task Force for the Diagnosis and Treatment of Pulmonary Hypertension of the European Society of Cardiology (ESC) and the European Respiratory Society (ERS): Endorsed by: Association for European Paediatric and Congenital Cardiology (AEPC), International Society for Heart and Lung Transplantation (ISHLT). Eur Respir J 2015; 46: 903-975. 
4 Gerges C, Gerges M, Lang MB, et al. Diastolic pulmonary vascular pressure gradient: a predictor of prognosis in "out-of-proportion" pulmonary hypertension. Chest 2013; 143: 758-766.

5 Gerges M, Gerges C, Pistritto AM, et al. Pulmonary hypertension in heart failure: epidemiology, right ventricular function and survival. Am J Respir Crit Care Med 2015; 192: 1234-1246.

6 Moher D, Liberati A, Tetzlaff J, et al. Preferred Reporting Items for Systematic Reviews and Meta-Analyses: The PRISMA Statement. PLoS Med 2009; 6: e1000097.

7 Miller WL, Grill DE, Borlaug BA. Clinical features, hemodynamics, and outcomes of pulmonary hypertension due to chronic heart failure with reduced ejection fraction: pulmonary hypertension and heart failure. JACC Heart Fail 2013; 1: 290-299.

8 Al-Naamani N, Preston IR, Paulus JK, et al. Pulmonary arterial capacitance is an important predictor of mortality in heart failure with a preserved ejection fraction. JACC Heart Fail 2015; 3: 467-474.

9 Palazzini M, Dardi F, Manes A, et al. Pulmonary hypertension due to left heart disease: analysis of survival according to the haemodynamic classification of the 2015 ESC/ERS guidelines and insights for future changes. Eur $J$ Heart Fail 2017; in press [https://doi.org/10.1002/ejhf.860].

10 Dragu R, Rispler S, Habib M, et al. Pulmonary arterial capacitance in patients with heart failure and reactive pulmonary hypertension. Eur J Heart Fail 2015; 17: 74-80.

11 Tampakakis E, Leary PJ, Selby VN, et al. The diastolic pulmonary gradient does not predict survival in patients with pulmonary hypertension due to left heart disease. JACC Heart Fail 2015; 3: 9-16.

12 O'Sullivan CJ, Wenaweser P, Ceylan O, et al. Effect of pulmonary hypertension hemodynamic presentation on clinical outcomes in patients with severe symptomatic aortic valve stenosis undergoing transcatheter aortic valve implantation: insights from the new proposed pulmonary hypertension classification. Circ Cardiovasc Interv 2015; 8: e002358.

13 Assad TR, Hemnes AR, Larkin EK, et al. Clinical and biological insights into combined post- and pre-capillary pulmonary hypertension. J Am Coll Cardiol 2016; 68: 2525-2536.

14 Brunner NW, Yue SF, Stub D, et al. The prognostic importance of the diastolic pulmonary gradient, transpulmonary gradient, and pulmonary vascular resistance in patients undergoing transcatheter aortic valve replacement. Cath Cardiovasc Interv 2017; 90: 1185-1191.

15 Duval S, Tweedie R. Trim and fill: A simple funnel-plot-based method of testing and adjusting for publication bias in meta-analysis. Biometrics 2000; 56: 455-463.

16 Phan K, Tian DH, Cao C, et al. Systematic review and meta-analysis: techniques and a guide for the academic surgeon. Ann Cardiothorac Surg 2015; 4: 112-122.

17 Houston BA, Tedford RJ. What we talk about when we talk about the wedge pressure. Circ Heart Fail 2017; 10: e004450.

18 Tedford RJ, Hassoun PM, Mathai SC, et al. Pulmonary capillary wedge pressure augments right ventricular pulsatile loading. Circulation 2012; 125: 289-297. 